\title{
ON INTERNAL CONSTRAINTS IN CONTINUUM MECHANICS
}

\author{
K. R. RAJAGOPAL AND GIUSEPPE SACCOMANDI
}

Received 18 June 2005; Accepted 22 August 2005

Dedicated to Professor Piero Villaggio on the occasion of his seventieth birthday

In classical particle mechanics, it is well understood that while working with nonholonomic and nonideal constraints, one cannot expect that the constraint be workless. It is curious that in continuum mechanics, however, the implications of the result in classical mechanics have not been clearly understood. In this paper, we show that in dealing with the response of dissipative systems, one cannot require that constraints do no work or ignore the fact that the material response functions depend on the constraint reaction. An example of this is the viscosity of a fluid depending on the pressure.

Copyright (c) 2006 K. R. Rajagopal and G. Saccomandi. This is an open access article distributed under the Creative Commons Attribution License, which permits unrestricted use, distribution, and reproduction in any medium, provided the original work is properly cited.

\section{Introduction}

Many of the theories that have been developed to describe the nonlinear response of continua had to take into consideration that the response of the body can be constrained. For example, the body under consideration might be incompressible or inextensible. While no real body is perfectly incompressible or inextensible, its response might be such that it can be approximated as meeting such a constraint for a large class of processes. For example, liquids or solids such as rubber, can, under normal operating conditions, be modelled as incompressible bodies. Idealizing such bodies as incompressible bodies accords a tremendous simplification in analyzing their thermomechanical response by virtue of which one can establish analytical closed-form solutions for a large class of problems that have scientific and technological relevance.

A variety of constraints can be enforced on a body. Here, we will consider purely mechanical constraints, in fact purely kinematical constraints such as incompressibility or inextensibility. A constraint such as incompressibility belongs to the class of constraints called elastic constraints (see [21]) which are defined through

$$
\varphi(\mathbf{F})=0
$$

Hindawi Publishing Corporation

Differential Equations and Nonlinear Mechanics

Volume 2006, Article ID 18572, Pages 1-12

DOI 10.1155/DENM/2006/18572 
where $\mathbf{F}$ is the deformation gradient. The special case of incompressibility takes the form

$$
\operatorname{det}(\mathbf{F})=1 \text {. }
$$

However, the same can be expressed as

$$
\operatorname{div}(\mathbf{v})=\operatorname{tr}(\mathbf{D})=0,
$$

provided that $\mathbf{F}$ is differentiable with respect to time. (Here $\mathbf{v}$ is the velocity field and $\mathbf{D}$ is the symmetric part of the velocity gradient $\mathbf{L}=\operatorname{grad}(\mathbf{v})$.) Now, the condition $\operatorname{tr}(\mathbf{D})=0$ is a special case of

$$
\psi(\mathbf{D})=0 .
$$

Because of (1.1) or (1.4), the stress in a body can no longer be specified only in terms of the deformation, and a dynamical characterization of the constraint is needed; that is, it is necessary to specify which kind of internal forces are needed to enforce the constraint. For elastic constraints, it is usual to require that the such constraint forces do no work. For general constraints such as (1.4), the situation is more complex and only recently, it has been shown by Rajagopal and Srinivasa [18] that the usual procedure of requiring that the constraint forces do no work, when the constraint is expressed in the form (1.1), is not valid when general constraints of the form (1.4) are required.

Great care has to be exercised when requiring what one means by the constraint force not doing work. For instance, if the constraint is that a block should move on a smooth horizontal surface, the constraint reaction is restricted to the force normal to the surface and this force does no work. However, if the constraint is that the block move on a plane rough surface, then while the force normal to the surface does no work, the forces due to friction that act tangential to the surface do work and we cannot forget the fact that this frictional force is also a consequence of the constraint and is a manifestation of the roughness of the surface. Also, we have to be more careful of what we require; whether we require that the normal forces do no work (which is trivially met in our case) or whether we should require that the constraint forces have no influence whatsoever on the work done.

It is well known that in the field of classical dynamics of particles and rigid bodies, elastic constraints correspond to holonomic constraints, whereas a constraint such as (1.4) corresponds to a nonholonomic constraint. When we deal with nonlinear nonholonomic constraints, in general, it is not possible to require that the constraints do no work. To be more precise, when the constraints are acatastic [16] - that is, linear, but not integrable equations in the time derivatives of the generalized coordinates - it is still possible to speak of ideal (in the sense of workless) constraints. Therefore, in the acatastic case, it is possible to generalize the usual Lagrange equations as has been done by several authors [16]. On the other hand, in the case of general nonlinear nonholonomic constraints, there has been, and there still is, a considerable amount of uncertainty and disagreement concerning the choice of the reaction forces since it is not possible to speak of ideal constraints and it is not clear as to how the basic equations of motion can be obtained, that is, the equations containing only the kinematical unknowns, in a systematic way. (See the 
book by Papastavridis [16] for a detailed bibliography on this issue). The status of nonideal constraints is so confused that the usual attitude is to bury one's head in the sand. For example, Goldstein [8] observes the following:

We now restrict ourselves to systems for which the net virtual work of forces of constraints is zero. We have seen that this condition holds true for rigid bodies and it is valid for a large number of other constraints. Thus, if a particle is constrained to move on a surface, the force of the constraint is perpendicular to the surface, while the virtual displacement must be tangent to it, and hence the virtual work vanishes. This is no longer true if sliding friction forces are present, and we must exclude such systems from our formulation. The restriction is not usually happening since friction is usually a macroscopic phenomenon.

Unfortunately, in many problems of interest, not only can the friction forces not be neglected, they essentially determine the quintessential features of the response characteristic of bodies, and thus one does not have the luxury of ignoring the work done due to the frictional forces.

In 1829, Gauss [7] offered the following alternate requirement to the assumption that constraint forces do no work:

The motion of a system of material points connected together in any manner whatsoever, whose motions are modified by any external restraints whatsoever, proceeds in every instance in the greatest possible accordance with free motion, or under the least possible constraint;

that is, the least possible force that is required to maintain the constraint is the desired constraint force, according to Gauss. He however remains silent as to whether this lest force that maintains the constraint does work or is workless. Thus, it is fully consistent within such a requirement that constraint force do work. O'Reilly and Srinivasa [15] have considered the consequences of Gauss' criterion for a large class of constraints. They provide a geometric interpretation for the constraints with reaction force being normal to the constraints surface. They also do not require that the constraints ought to be workless. (About this point see also [23] for an alternative but related treatment).

When we consider a body, say a block, moving on a planar rough horizontal surface, it is first necessary to decide what one understands by the constraint force. In this case, the normal reaction force is denoted by $\mathbf{N}$, and the horizontal force (the friction) by using the classical dynamical Coulombs law is given by $\mu \mathbf{N}$ (where $\mu$ is the dynamic friction coefficient). Both the forces $\mathbf{N}$ and $\mu \mathbf{N}$ are a consequence of the body being constrained to move on a rough planar surface. To merely give $\mathbf{N}$ the status of the constraint reaction and then to require that it do no work is a trivial requirement as the part of the constraint reaction that is chosen is automatically normal to the constraint manifold, and thus can do no work as the body moves on the constraint manifold. One cannot afford to forget that the purpose of mathematics is to render with clarity and precision the ideas that are expressed in terms of words, in the present case, the body is constrained to move on a rough planar surface. Furthermore, even if one chooses to take the narrow viewpoint that $\mathbf{N}$ is the only constraint force, we cannot fail to recognize that $\mathbf{N}$ influences the work that is done. In fact, its magnitude determines precisely the work that is done. 
In their influential tome on nonlinear mechanics [22], Truesdell and Noll observe that

the stress $\mathbf{T}$ at time $t$ is determined by the history $\mathbf{F}^{(t)}(s)$ of the deformation gradient only to within a stress $\mathbf{N}$ that does no work in any motion satisfying the constraints.

This seems to suggest with unmistakable clarity that for viscous fluids or non-Newtonian fluids that have memory, we have to require that constraint forces, and this whatever the nature of the constraint, have to do no work in any admissible motion, that is, in any motion satisfying the constraint itself. Truesdell and Noll [22] are neither stating that only linear constraints in $\mathbf{D}$ will be considered, nor are they restricting their discussion to elastic bodies though they discuss elastic constraints. They are very clearly considering bodies whose stress is determined by the history of the deformation gradient and they evidently include numerous classes of dissipative bodies. Our earlier discussion obviously makes evident why such a position is too special when we consider dissipative materials. While speaking of reactions forces (i.e., constraint forces), Truesdell and Noll [22] allow that "more complicated systems of forces might be found" than the system that satisfies the assumption of worklessness; however this workless system seem to be the "simplest imaginable to suffice to maintain the constraint." There seems to be a clear connection being made here that the simplest system of forces that is required to maintain the constraint is workless. This is clearly incorrect for nonholonomic systems in particle and rigid body dynamics and also with regard to the response of dissipative continua. Surprisingly, there is no mention in Truesdell and Noll of Gauss' important contribution to the theory of constraints where the requirement of the least force necessary to maintain the constraint was first articulated.

\section{Constraints in dynamics}

The simplest model of classical mechanics is the material point, here we will use this simple model to clarify some trivial but often misunderstood fact about constraints and reaction forces.

Let us consider a point mass $E$, at the point $\mathbf{P} \in \mathbb{R}^{3}$ at time $t$, of mass $m$. If $E$ is unconstrained, to determine its motion in a given reference system we must know for any instant $t$ the vector $\mathbf{O P}(t)=x(t) \mathbf{i}+y(t) \mathbf{j}+z(t) \mathbf{k}$ and to this end, we must use Newton's second law:

$$
m \mathbf{a}=\mathbf{f}(\mathbf{O P}, \mathbf{v}, t)
$$

In classical mechanics, the assumption of determinism says that the Cauchy problem obtained, considering (2.1) subject the initial conditions $\mathbf{O P}(0)=\mathbf{O P}_{0}$ and $\mathbf{v}(0)=\mathbf{v}_{0}$, must have only one solution. This means that once the constitutive relation for the force law is given and some basic smoothness assumptions are satisfied, it is possible at any $t>0$ to determine the unique motion, $\mathbf{O P}\left(\mathbf{O P}_{0}, \mathbf{v}_{0} ; t\right)$, of the element $E$. A priori there are no restrictions about the constitutive equation for the force beside the smoothness requirements, therefore $\mathbf{f}$ is an arbitrary element of a vectorial space $\mathbb{V}$.

Now, to simplify the discussion, let $E$ be constrained by a single constraint that is holonomic, that is, given by an equation $\varphi(x, y, z ; t)=0$. In this case the set of the possible positions (i.e., compatible with the constraint) for $E$ is a proper submanifold $\mathscr{P}_{t} \subset \mathbb{R}^{3}$. For 
any point $P \in \mathscr{P}_{t}$ and any time $t$, we may compute the tangent space $\mathscr{T}_{P, t}$ of $\mathscr{P}_{t}$ at $P$ to find the set $\mathscr{V} \operatorname{ir}(P, t)$ of the virtual velocities of $E$. The set of the possible velocities is denoted as $\mathscr{V}(P, t)$ and the elements of this set are the vectors

$$
\mathbf{v}=\mathbf{v}_{t}+\mathbf{v}_{n}(P, t)
$$

where $\mathbf{v}_{t}$ is an arbitrary element of $\mathscr{T}_{P, t}$, whereas $\mathbf{v}_{n}(P, t)$ is uniquely determined by $P$ and $t$ and clearly $\mathbf{v}_{t} \perp \mathbf{v}_{n}$. Moreover we denote by $\mathscr{A}(P, \mathbf{v}, t)$ the set of the admissible acceleration vectors. For any $P \in \mathscr{P}_{t}$ and $\mathbf{v} \in \mathscr{V}(P, t)$, if $\mathbf{a} \in \mathscr{A}(P, \mathbf{v}, t)$, again a decomposition similar to (2.2) is possible, that is,

$$
\mathbf{a}=\mathbf{a}_{t}+\mathbf{a}_{n}(\mathbf{v}, P, t)
$$

where $\mathbf{a}_{t}$ is an arbitrary element of $\mathscr{T}_{P, t}$, whereas $\mathbf{a}_{n}(\mathbf{v}, P, t)$ is uniquely determined by $\mathbf{v}$, $P$, and $t$.

In this framework, the need to introduce a reaction force arises from a purely algebraic stand point. Indeed, in the constrained case, the basic equation (2.1) cannot be verified for any general constitutive relation for the forces (i.e., independent of the material point and therefore of the constraint that has been introduced) because, generally speaking, $\mathbb{V} \nsubseteq \mathscr{A}(P, \mathbf{v}, t)$. The reaction force $\mathbf{r}$ is therefore introduced such that $(2.1)$ is modified as

$$
m \mathbf{a}=\mathbf{f}(\mathbf{O P}, \mathbf{v}, t)+\mathbf{r}
$$

and now $\mathbf{f}(\mathbf{O P}, \mathbf{v}, t)+\mathbf{r} \in \mathscr{A}(P, \mathbf{v}, t)$. Obviously there is an infinity of vectors $\mathbf{r}$ such that (2.4) defines a vector $\mathbf{a} \in \mathscr{A}(P, \mathbf{v}, t)$ and this goes against the requirement of determinism. There is no way out of this indeterminism if some restriction on $\mathbf{r}$ is not introduced (this restriction is usually called the dynamical characterization of the constraint).

We observe that, to the best of our knowledge, many texts devoted to classical mechanics give several explicit examples of dynamical characterizations of the constraints but do not give a rigorous definition of such characterization. A possible definition, proposed by Valente and Benevenuti [24], is the following one: given any value for $P, \mathbf{v}, t$ such that $P \in \mathscr{P}_{t}$ and $\mathbf{v} \in \mathscr{V}(P, t)$, a set of vectors $\mathscr{R}(P, \mathbf{v}, t) \subset V^{3}$ is an admissible reaction force if

(i) for all $\mathbf{f} \in V^{3}$, there exists a unique value of $\mathbf{r}(\mathbf{f}) \in \mathscr{R}(P, \mathbf{v}, t)$ such that $\mathbf{f}+\mathbf{r}(\mathbf{f}) \in$ $\mathscr{A}(P, \mathbf{v}, t)$

(ii) for all $\mathbf{a} \in \mathscr{A}(P, \mathbf{v}, t)$, there exists at least a force field $\mathbf{f} \in V^{3}$ such that $\mathbf{f}+\mathbf{r}(\mathbf{f})=\mathbf{a}$.

It is important to point out that following this definition, the dynamical characterization of the constraint is a map that to any possible choice of $P, \mathbf{v}, t$ associates a set of admissible constraints $\mathscr{R}(P, \mathbf{v}, t)$. Once such a characterization is given, the property (i) ensures that there exists one function $\mathbf{r}=\mathbf{r}^{*}(P, \mathbf{v}, t, f)$ such that all the motions defined by (2.4) are compatible with the constraint. Since $\mathbf{f}+\mathbf{r}^{*} \in \mathscr{A}(P, \mathbf{v}, t)$ and $\mathbf{r}^{*}$ is unique, the requirement of determinism will hold (under suitable smoothness assumptions).

Now, that we have given a clear definition of what we mean for a dynamical characterization, it is possible to use a very simple geometrical argument to understand in an 
intuitive way what is going on. Equation (2.4) may be always decomposed:

$$
\begin{gathered}
\mathbf{r}_{t}=m \mathbf{a}_{t}-\mathbf{f}_{t}(P, \mathbf{v}, t), \\
\mathbf{r}_{n}=m \mathbf{a}_{n}(P, \mathbf{v}, \mathbf{t})-\mathbf{f}_{n}(P, \mathbf{v}, t),
\end{gathered}
$$

and it is therefore clear that once that the external force is assigned, the condition $\mathbf{a} \in$ $\mathscr{A}(P, \mathbf{v}, t)$ does not impose any restrictions to $\mathbf{r}_{t}$, whereas it determines completely and uniquely $\mathbf{r}_{n}$. In this framework, the choice $\mathbf{r}_{t}=0$ and $\mathbf{r}_{n}$ defined by the second formula of (2.5) is the simplest choice that allows one to solve the nonuniqueness problem and the determination of the reaction force. We point out again that the choice $\mathbf{r}_{t}=0$ is here introduced only on the basis of geometrical considerations without the introduction of a dynamical characterization.

The simple geometrical characterization, presented in this section, allows us to clarify two important points. First we clarify why ideal constraints are the simplest imaginable to suffice to maintain the constraint. Then it is also clear why an ideal constraint may be very far away from physical reality; they may be introduced on the basis of pure geometrical reasons without any constitutive requirement.

On the other hand, from (2.5), it is also possible to understand why the classical theory of friction proposed by Coulomb and Morin (see, e.g., [4]) is a basic one. Indeed it is now clear that because the $\mathbf{r}_{n}$ is completely and uniquely determined, the problem of any theory of friction is to give a constitutive equation for $\mathbf{r}_{t}$. A constitutive equation must determine only a unique value for any external force $\mathbf{f}$ and therefore this implies that $\mathbf{r}_{t}=\varphi\left(\mathbf{O P}, \mathbf{v}, t, \mathbf{r}_{n}\right)$. Because we know a priori from knowledge of the constraint in the direction of $\mathbf{r}_{t}$, the Coulomb-Morin law $\left|\mathbf{r}_{t}\right|=\mu_{D}\left|\mathbf{r}_{n}\right|$ is the simplest linear relationship that is in accord with the general principles introduced here.

It is important to note that our discussion may be easily extended to nonholonomic nonlinear constraints. For example, a similar decomposition as in (2.5) is always possible, but now we have a situation wherein the normal direction to the surface described by the constraint is no more the normal to the velocities and immediately follows that the minimal reaction forces, generally speaking, in this case do work.

We wish to point out that the geometric argument presented here also works in the case of an $\mathrm{N}$-degree of freedom. The extension to any degree of freedom of (2.5) has been done, for example, by Udwadia and Kalaba [23] using a formalism based on the MoorePenrose pseudoinverse. We recall that the pseudoinverse is unique only in the sense of quadratic minimization and therefore we go back to the idea introduced by Gauss.

\section{Constraints in continua: incompressible bodies with pressure-dependent material moduli}

We now discuss the response of constrained bodies, continua, which undergo processes wherein the constraint forces do work, as the processes under consideration are dissipative; while the constraint in itself is what is called by Truesdell [21] an elastic constraint as it falls under the category delineated by (1.1). Here, as discussed in the introduction, we have to take due care of what we mean by the constraint force (or constraint response). 
The constraint under consideration is the most commonly used one, namely that of incompressibility, that is, the constraint (1.2) which can be expressed as (1.3).

Our discussion here follows in large part that by Rajagopal and Srinivasa [18]. The normal procedure that is used to determine the constraint response is (see [21]) to split the Cauchy stress tensor $\mathbf{T}$ additively as

$$
\mathbf{T}=\mathbf{T}_{C}+\mathbf{T}_{E}
$$

where $\mathbf{T}_{C}$ is the constraint stress and $\mathbf{T}_{E}$ is the extra stress that is constitutively determined. One can then assume that $\mathbf{T}_{E}$ does not depend on the quantities that appear in $\mathbf{T}_{C}$ and requiring that $\mathbf{T}_{C}$ does no work, that is, that

$$
\mathbf{T}_{C} \cdot \mathbf{D}=0 \quad \text { whenever } \operatorname{tr}(\mathbf{D})=\mathbf{I} \cdot \mathbf{D}=0 .
$$

This immediately leads to

$$
\mathbf{T}_{C}=-\phi \mathbf{I},
$$

and using the usual convention of labelling $\phi$ by $-p$, we find that

$$
\mathbf{T}=-p \mathbf{I}+\mathbf{T}_{E}
$$

where $\mathbf{T}_{E}$ is not dependent on the Lagrange multiplier $p$. This procedure would however fail if the constraint was nonlinear in $\mathbf{D}$ (see [18]). Antman [2] (see also [3]) recognized that requiring that the extra stress $\mathbf{T}_{E}$ does not depend on the Lagrange multiplier was too restrictive. He however did not question the splitting of the stress as in (3.1) or the appropriateness of the assumption that the constraint stress ought to be workless.

There is a considerable body of literature concerning fluids that could be approximated as incompressible while their material properties such as viscosity or relaxation time could depend upon the pressure. Stokes [19] recognized that the viscosity of a fluid could depend on the pressure. Andrade [1] gave an explicit form for the variation of the viscosity with density temperature and pressure:

$$
\mu=\tilde{\mu}(\rho, \theta, p)=A \rho^{1 / 2} \exp \left[\left(p+\rho^{2} \nu\right) \frac{s}{\theta}\right],
$$

where $A, v$, and $s$ are positive constants. Extensive studies have been carried out to determine the viscosity of liquids with pressure (an exhaustive documentation of the work prior to 1930 can be found in the magisterial book by Bridgman [5]). More recent experimental results can be found in $[6,10,12,13]$. While (3.5) indicates that the viscosity depends on the density and pressure for many liquids, the variation of the density is of the order of a few percent while the pressure changes by several orders of magnitude leading to a corresponding variation in viscosity by several orders of magnitude. Thus, it would be reasonable for such liquids to be considered incompressible with the viscosity depending on the pressure. In certain applications such as elastohydrodynamics, this is precisely the situation. While the density varies ever so slightly, the fluids viscosity varies by several orders of magnitude. The viscosity is in fact assumed to vary exponentially with the pressure (see [20]). 
In continuum mechanics, there is a large body of literature concerning internal constraints but, to the best of our knowledge, only in the recent papers by Rajagopal and coworkers has a more general theory than the one of workless internal constraints been developed and have problems been solved in some detail. The main advantage of the theory is the use of a direct geometrical approach in introducing internal constraints. Here, we wish to point out that the basic construction of this theory follows from a parallel argument from the ideas discussed in the previous section concerning classical dynamics.

Consider a body that occupies a configuration $\mathscr{S}_{t}$ at a given time $t$. Let the mass density be $\rho(\mathbf{x}, t)$ and let the position, velocity, and acceleration of the particle of the body be $\mathbf{x}(\mathbf{X}, t), \mathbf{v}(\mathbf{x}, t), \mathbf{a}(\mathbf{x}, t)$, respectively. The laws for the mechanical response of continua are the balance of mass

$$
\frac{d \rho}{d t}+\rho \operatorname{div}(\mathbf{v})=0
$$

and the balance of linear momentum

$$
\rho \mathbf{a}=\operatorname{div}(\mathbf{T})+\rho \mathbf{b}
$$

where $d(\cdot) / d t$ is the material time derivative and $\mathbf{b}$ is the specific body force. In the absence of body couples, the balance of angular momentum requires that the Cauchy stress tensor is symmetric; that is, $\mathbf{T}=\mathbf{T}^{T}$. The notation $\operatorname{div}(\cdot)$ represent the divergence with respect to spatial coordinates.

It is necessary to add to (3.6) and (3.7) a constitutive equation for the Cauchy stress tensor, and it is typical that the stress tensor is specified as a function of the history of the deformation gradient $\mathbf{F}^{t}(s)=\mathbf{F}(t-s),(\mathbf{F}=\partial \mathbf{x} / \partial \mathbf{X})$. For example, in elasticity, a constitutive equation is a mapping:

$$
\widetilde{\mathbf{T}}: \mathscr{D} \longrightarrow \mathscr{C}, \quad \mathrm{T}=\tilde{\mathrm{T}}(\mathbf{F})
$$

In the case of unconstrained elastic bodies, $\mathscr{D}$ is the collection of second-order tensors with positive determinants (a collection denoted with $\mathrm{Lin}^{+}$).

The mathematical formulation of an internal constraint is usually an equation of the form (1.1). Sometimes this definition is given in a more abstract setting concerning a connected $C^{1}$-manifold $M \subset \operatorname{Lin}^{+}$such that $\mathbf{I} \in \mathcal{M}$, and requiring that $\mathcal{M}$ be invariant under changes in frame, the usual objectivity requirement that may also be translated into

$$
\hat{\varphi}(\mathbf{C})=0
$$

where $\mathbf{C}=\mathbf{F}^{T} \mathbf{F}$. Equation (3.9) introduces a restriction on the domain of the mapping (3.8).

On the other hand, it is possible also to consider a constraint of the form (1.3). Beside the usual incompressibility constraint, we note that Green et al. [9] have considered internal constraints that depend not only on the gradient of deformation but also on the 
stretching tensor, introducing the following relation:

$$
\mathbf{A}(\mathbf{C}) \cdot \mathbf{D}+a(\mathbf{C})=0
$$

where $\mathbf{A}$ is some tensor and $a$ is some scalar. The functional form (3.10) is dictated by the fact that only for a constraint linear in $\mathbf{D}$ is it possible to characterize the reaction stress as workless. Equation (3.10) is the continuum mechanics counterpart of acatastic constraints. To the best of our knowledge, it is only in [18] that a full nonlinear anholonomic constraint for continua has been considered.

It is clear that the constraint is not only a restriction on the domain $\mathscr{D}$ of the mapping (3.8) but also on $\mathbf{x}(\mathbf{X}, t)$ and all the kinematical quantities of interest. To investigate in detail the situation, let us consider a general constraint of the form (1.3). First of all, by differentiation of (1.4) with respect to time, we obtain

$$
\frac{\partial \psi}{\partial \mathrm{D}} \cdot \frac{\partial \mathrm{D}}{\partial t}=0
$$

The nature of internal constraints such as (1.3) is clear only in an appropriate tensorial space, and as the balance of linear momentum is a vectorial relationship, it is quite hard to understand the restriction imposed by (3.11) on (3.7) within the context of a simple geometrical picture. In order to gain some clarity concerning this situation, we take the gradient of (3.7), thereby obtaining

$$
\frac{\partial \mathbf{L}}{\partial t}+\operatorname{grad}(\mathbf{L v})=\operatorname{grad}\left(\frac{1}{\rho} \operatorname{div}(\mathbf{T})+\mathbf{b}\right) .
$$

Now, it is clear that because $\mathbf{T}$ and $\mathbf{b}$ in (3.12) may be, in principle, arbitrary (obviously the stress tensor has to satisfy some general requirements of invariance but here these requirements are of no interest) and therefore treating this equation as a simple algebraic relation, it is possible to choose the right-hand side of (3.12) in such a way that the lefthand side does not satisfy the constraint. To see this we introduce $\mathbf{N}=\partial \psi / \partial \mathbf{D}$ and the tensor $\mathbf{M}$ such that $\mathbf{M} \perp \mathbf{N}$. It follows from (3.12) on using (3.11) that

$$
\begin{gathered}
\mathbf{N} \cdot \operatorname{grad}(\mathbf{L v})=\mathbf{N} \cdot \operatorname{grad}\left(\frac{1}{\rho} \operatorname{div}(\mathbf{T})+\mathbf{b}\right), \\
\mathbf{M} \cdot \frac{\partial \mathbf{L}}{\partial t}+\mathbf{M} \cdot \operatorname{grad}(\mathbf{L v})=\mathbf{M} \cdot \operatorname{grad}\left(\frac{1}{\rho} \operatorname{div}(\mathbf{T})+\mathbf{b}\right) .
\end{gathered}
$$

It is clear that the first formula of (3.13) cannot generally be satisfied for any choice of $\mathbf{T}$ and $\mathbf{b}$. Whereas the presence of the first time derivatives of the velocity field in the second formula of (3.13) allows us to ensure that this equation may be satisfied for any choice of $\mathbf{T}$ and $\mathbf{b}$. Therefore, as we do in classical mechanics, we need to introduce a reaction stress ( in reality, we need a reaction force, but because we are considering internal forces, it is natural to require that this force be related to a stress).

Once again let us restrict our attention to incompressibility, that is,

$$
\mathbf{N}=\mathbf{I}, \quad \mathbf{M}=\{\mathbf{D}: \operatorname{trace}(\mathbf{D})=0\} .
$$


If we introduce the reaction stress as $-p \mathbf{I}$, this is sufficient to meet (3.13). Indeed, in this case, the equation reduces to

$$
\mathbf{I} \cdot \operatorname{grad}(\mathbf{L v})=-\mathbf{I} \cdot \operatorname{grad}(\operatorname{grad}(p))+\mathbf{I} \cdot \operatorname{grad}\left(\frac{1}{\rho} \operatorname{div}(\mathbf{T})+\mathbf{b}\right)
$$

a second-order linear differential equation for the pressure. Therefore, for the ideal internal constraint of incompressibility, it is sufficient to meet (3.7) such that for any choice of $\mathbf{T}$ and $\mathbf{b}$ the acceleration a will always satisfy the prescription of the constraint. Here, we have not appealed to the constraint to be workless, rather we have automatically introduced a reaction stress that satisfies worklessness as a byproduct of geometrical considerations. The idea is to merely ensure that (3.13) can be met and the assumption that the reaction stress is $-p \mathbf{I}$ allows us to do this, that is, it is sufficient for meeting (3.13).

It is clear that when we have a full nonlinear constraint we will have difficulty in satisfying (3.13) with a workless constraint, but also in the special case of incompressibility, a more general theory may be developed. Let us consider the model

$$
\mathbf{T}=-p \mathbf{I}+2 \mu(p) \mathbf{D}
$$

Recently, there have been several studies concerning such a fluid from both a numerical and analytical perspectives (see $[11,14]$ ). We are not interested in discussing these results or the response characteristics of the fluid modelled by (3.16). The point that we are interested in making is that what is usually regarded as the constraint, response, $-p \mathbf{I}$, while in itself does no work, determines the work done, that is, the work done is given by

$$
\mathbf{T} \cdot \mathbf{D}=2 \mu(p) \mathbf{D} \cdot \mathbf{D} .
$$

More importantly, for models such as (3.16) in analogy with the example discussed in our introduction concerning the sliding block, we recognize that the shear stresses that would be introduced in a simple shear flow are also part of the constraint. Let us consider the simple shear flow between two parallel plates and let us suppose that the fluid is incompressible and modelled by (3.16). Further, let us suppose that gravity acts normal to the plates. Let us consider the counterpart of the classical Couette flow, and as is customary, let us seek a solution for the velocity field of the form

$$
\mathbf{v}=u(y) \mathbf{i}
$$

where $u$ is the component of the velocity in the $x$-coordinate direction. As we have gravity acting along the $y$-coordinate direction, we will seek a pressure field of the form

$$
p=p(y)
$$

It is trivial to show that

$$
p=p_{a}+\rho g y,
$$

where $p_{a}$ is the atmospheric pressure. We will not be interested in solving any boundary value problems here. It has been shown (see [17]) that pronounced boundary layers can 
develop due to the viscosity. Here, we are merely interested in clarifying what one means by a constraint reaction and whether this reaction does or does not do any work.

We notice that at any plane $y=$ constant, in addition to the normal force on the plane that corresponds to the weight of the fluid above, the shear stresses acting on the layer at $y=$ constant depend on the pressure, that is,

$$
T_{x y}=\mu(p) \frac{d u}{d y}
$$

Even if the viscosity of the fluid does not depend on the pressure, the shear stress $T_{x y}$ is not zero, but in this case there is seemingly no connection between the constraint reaction and the shear stress. However, when the viscosity depends on the pressure, we cannot clearly define what is meant by the constraint response, the spherical part of the form $-p \mathbf{I}$ and the extra stress $+2 \mu(p) \mathbf{D}$ are related. However, the extra stress having the specific form $2 \mu(p) \mathbf{D}$ is a consequence of the constraint. Thus, it is not that simple to dismiss the term as not being a part of the constraint response.

We hope that these examples are sufficient to explain why the theory of internal constraints as it stands in continuum mechanics needs to be reexamined carefully and a more general theory put into place.

\section{Acknowledgment}

The second author is partially supported by the Project "Problemi Matematici Non Lineari di Propagazione e Stabilità nei Modelli del Continuo", MURST Cofin 2003, and ex-60\% funds of the University of Lecce.

\section{References}

[1] E. C. Andrade, Viscosity of liquids, Nature 125 (1930), 309-310.

[2] S. S. Antman, Material constraints in continuum mechanics, Atti della Accademia Nazionale dei Lincei, Classe di Scienze Fisiche, Matematiche e Naturali 70 (1982), 256-264.

[3] S. S. Antman and R. S. Marlow, Material constraints, Lagrange multipliers, and compatibility. Applications to rod and shell theories, Archive for Rational Mechanics and Analysis 116 (1991), no. 3, 257-299.

[4] P. Appell, Traité de Mécanique Rationelle II, Gauthier-Villars, Paris, 1953.

[5] P. W. Bridgman, The Physics of High Pressure, MacMillan, New York, 1931.

[6] W. G. Cutler, R. H. McMickle, W. Webb, and R. W. Schiessler, Study of the compressions of several high molecular weight hydrocarbons, Journal of Chemical Physics 29 (1958), no. 4, 727-740.

[7] C. F. Gauss, On a new general principle of mechanics, Philosophical Magazine 8 (1830), 137-140, Translation of Über ein neues allegemeines Grundgesetz der Mechanik, J. reine angew. Math. 4 (1829) 232-235.

[8] H. Goldstein, Classical Mechanics, 2nd ed., Addison-Wesley Series in Physics, Addison-Wesley, Massachusetts, 1980.

[9] A. E. Green, P. M. Naghdi, and J. A. Trapp, Thermodynamics of a continuum with internal constraints, International Journal of Engineering Science 8 (1970), no. 11, 891-908.

[10] E. M. Griest, W. Webb, and R. W. Schiessler, Effect of pressure on viscosity of higher hydrocarbons and their mixtures, Journal of Chemical Physics 29 (1958), no. 4, 711-720.

[11] J. Hron, J. Málek, and K. R. Rajagopal, Simple flows of fluids with pressure-dependent viscosities, Proceedings of the Royal Society of London Series A 457 (2001), no. 2011, 1603-1622. 


\section{On internal constraints in continuum mechanics}

[12] K. L. Johnson and R. Cameron, Shear behaviour of elastohydrodynamic oil films at high rolling contact pressures, Proceedings of the Institution of Mechanical Engineers 182 (1967), 307-319.

[13] K. L. Johnson and J. A. Greenwood, Thermal analysis of an Eyring fluid in elastohydrodynamic traction, Wear 61 (1980), no. 2, 353-374.

[14] J. Málek, J. Nečas, and K. R. Rajagopal, Global analysis of the flows of fluids with pressuredependent viscosities, Archive for Rational Mechanics and Analysis 165 (2002), no. 3, 243-269.

[15] O. M. O'Reilly and A. R. Srinivasa, On the nature of constraint forces in dynamics, Proceedings of the Royal Society of London Series A 457 (2001), 1307-1313.

[16] J. G. Papastavridis, Analytical Mechanics, Oxford University Press, Oxford, 2002.

[17] K. R. Rajagopal, On implicit constitutive theories, Applications of Mathematics 48 (2003), no. 4, 279-319.

[18] K. R. Rajagopal and A. R. Srinivasa, On thermomechanical restrictions of continua, Proceedings of The Royal Society of London. Series A. Mathematical, Physical and Engineering Sciences $\mathbf{4 6 0}$ (2004), no. 2042, 631-651.

[19] G. G. Stokes, On the theories of internal friction of fluids in motion and of the equilibrium and motion of elastic solids, Transactions Cambridge Philosphical Society 8 (1845), 287-305.

[20] A. Z. Szeri, Fluid Film Lubrication: Theory and Design, Cambridge University Press, Cambridge, 1998.

[21] C. A. Truesdell III, A First Course in Rational Continuum Mechanics. Vol. 1. General Concepts, 2nd ed., Pure and Applied Mathematics, vol. 71, Academic Press, Massachusetts, 1991.

[22] C. A. Truesdell III and W. Noll, The Nonlinear Field Theories of Mechanics, 2nd ed., Springer, Berlin, 1992.

[23] F. E. Udwadia and R. E. Kalaba, What is the general form of the explicit equations of motion for constrained mechanical systems?, American Society of Mechanical Engineers. Transactions of the ASME. Journal of Applied Mechanics 69 (2002), no. 3, 335-339.

[24] C. Valente, Characterizing constraint stresses, Università degli Studi di Roma "La Sapienza". Dipartimento di Metodi e Modelli Matematici per le Scienze Applicate. Pubblicazioni (1984), no. 22, 43-59 (Italian).

K. R. Rajagopal: Department of Mechanical Engineering, Texas A \& M University, College Station, TX 77845, USA

E-mail address: krajagopal@mengr.tamu.edu

Giuseppe Saccomandi: Dipartimento di Ingegneria dell'Innovazione, Università degli Studi di Lecce, 73100 Lecce, Italy

E-mail address: giuseppe.saccomandi@unile.it 


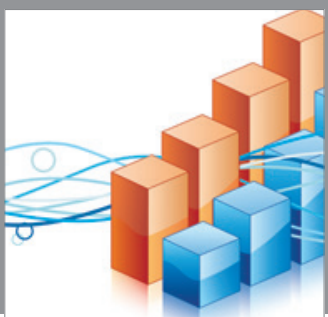

Advances in

Operations Research

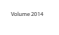

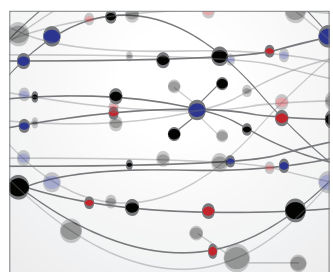

\section{The Scientific} World Journal
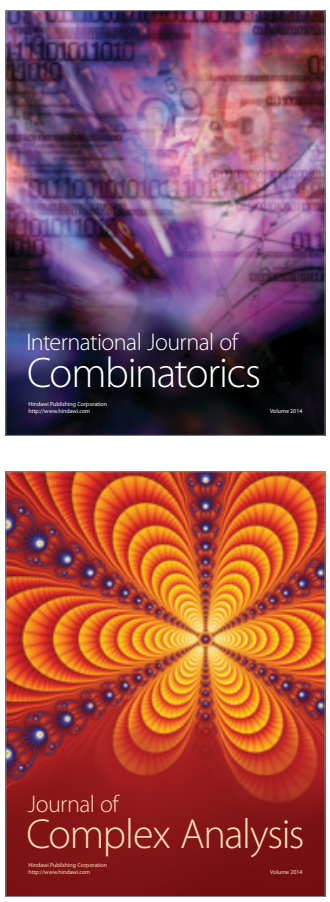

International Journal of

Mathematics and

Mathematical

Sciences
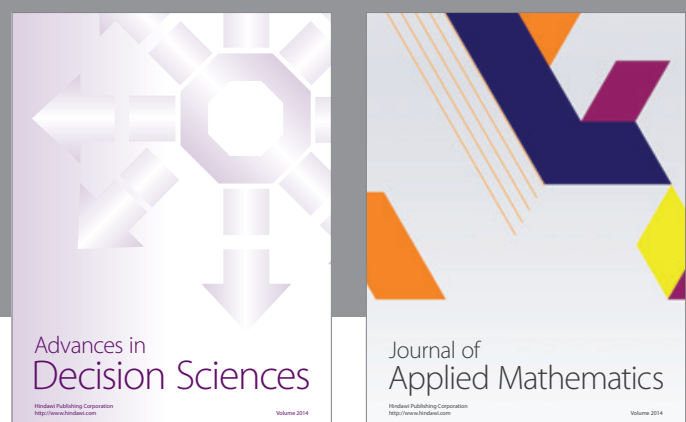

Journal of

Applied Mathematics
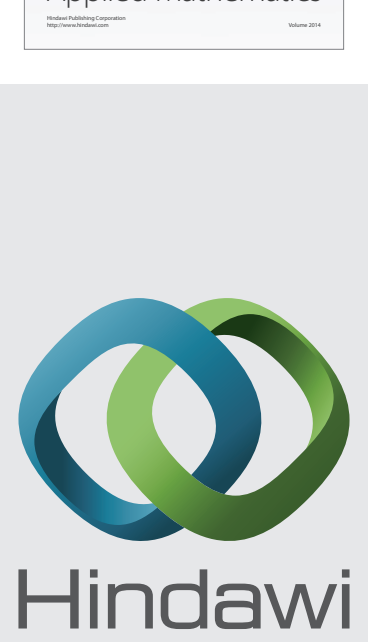

Submit your manuscripts at http://www.hindawi.com
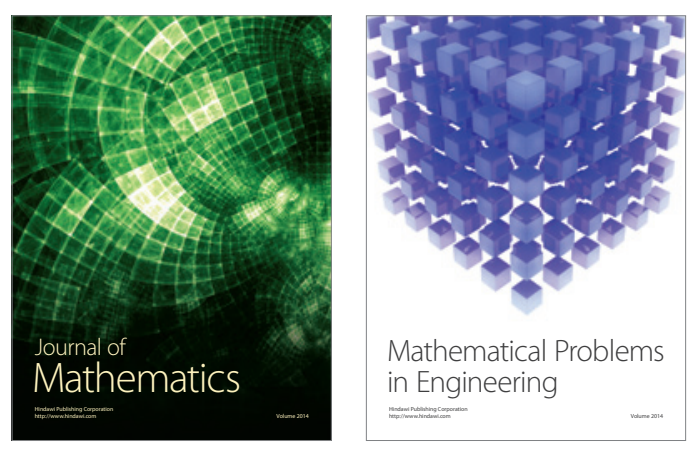

Mathematical Problems in Engineering
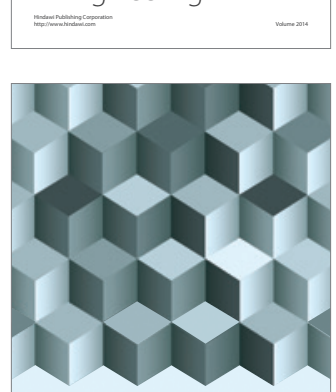

Journal of

Function Spaces
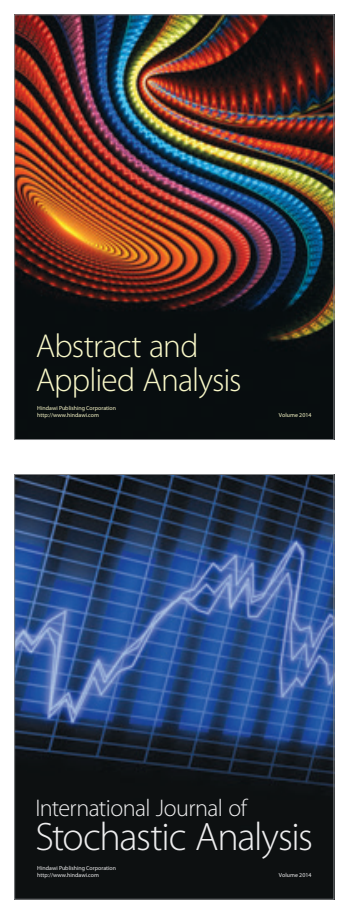

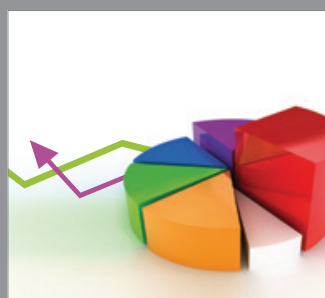

ournal of

Probability and Statistics

Promensencen
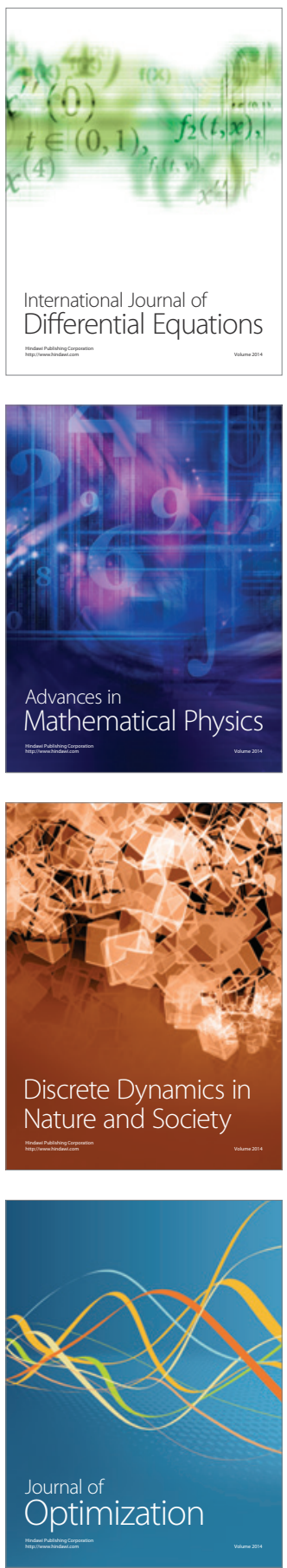